supplies of guanethidine; and Merck Sharp and Dohme Ltd for a mobile screening unit, funds for its staffing, and supplies of methyldopa.

\section{References}

1 Medical Research Council Working Party. MRC trial of treatment of mild hypertension: principal results. Br Med f 1985;291:97-104.

2 Kannel WB, Sorlie P, Gordon T. Labile hypertension: a faulty concept? The Framingham study. Circulation 1980;61:1183-7.

3 Cruickshank JM, Thorp JM, Zacharias FJ. Benefits and potential harm of lowering high blood pressure. Lancet 1987;i:581-4.

4 Berglund G, Samuelsson O. Lowered blood pressure and the J-shaped curve. Lancet 1987;i:1154-5.
5 IPPPSH Collaborative Group. Cardiovascular risk and risk factors in a randomised trial of treatment based on the beta-blocker oxprenolol: the international prospective primary prevention study in on the beta-blocker oxprenolol: the international prospective

6 Cruickshank JM. MRC tria

7 Medical Research Council Working Party. Coronary heart disease in the MRC trial of treatment of mild hypertension. Br Heart $\mathcal{F}$ 1988;59:364-78.

8 Medical Research Council Working Party on Mild to Moderate Hypertension. Adverse reactions to bendrofluazide and propranolol for the treatment of mild hypertension. Lancet 1981;ii:539-43.

9 Medical Research Council Working Party. Comparison of the antihypertensive efficacy and adverse reactions to two doses of (i) bendrofluazide and (ii) hydrochlorothiazide, and the effect of potassium supplementation on the hypotensive action of bendrofluazide. 7 Clin Pharmacol $1987 ; 27: 271-7$.

(Accepted 12 February 1988)

\title{
Protective efficacy of a whole cell pertussis vaccine
}

\author{
MARGARETA BLENNOW, PATRICK OLIN, MARTA GRANSTRÖM, ROGER H BERNIER
}

\begin{abstract}
A trial of the efficacy of a plain whole cell pertussis vaccine was conducted in Sweden. In this non-blinded trial 525 infants aged 2 months who were born on days with an even number received three doses of vaccine one month apart and 615 infants of the same age who were born on days with an odd number were enrolled as controls. During the $\mathbf{1 8}$ months of follow up there were 55 cases of pertussis. The attack rate was $1.5 \%(8 / 525)$ among the vaccinated children and $7 \cdot 6 \%(47 / 615)$ among the unvaccinated children $(p<0.001)$. The estimated efficacy of the vaccine was $80 \%$ ( $95 \%$ confidence interval 58 to 90 ).

The estimated efficacy of pertussis vaccine was similar to that observed in British trials over 30 years ago.
\end{abstract}

\section{Introduction}

A national immunisation programme against pertussis was introduced in Sweden, as in many other countries, in the mid-1950s. After years of the incidence of the disease decreasing epidemics of pertussis started to occur in the 1970s. This resurgence was associated with a decreased efficacy of the vaccine and together with a growing concern about the safety of the vaccine led to the decision in 1979 to stop the routine use and production of pertussis vaccine. A plain whole cell vaccine (Pertussis Vaccine Wellcome; Wellcome) was imported from the United Kingdom in 1981 for limited use in children with cystic fibrosis and other conditions of high risk. ${ }^{12}$

The possibility that a further increase in the incidence of the disease would necessitate reintroducing general vaccination prompted further studies of Wellcome's product. Our study was started in 1982 to collect information on the antibody response and adverse

Karolinska Institute, Department of Paediatrics, Sachs's Children's Hospital, and Department of Vaccine Production, National Bacteriological Laboratory, Stockholm, Sweden

MARGARETA BLENNOW, MD, paediatrician

PATRICK OLIN, MD, associate professor

MARTA GRANSTRÖM, MD, associate professor

Division of Immunization, Centers for Disease Control, Atlanta, Georgia 30333, United States

ROGER H BERNIER, MPH, PHD, chief, epidemiology research section

Correspondence to: $\operatorname{Dr} M$ Blennow, Department of Vaccine Production, National Bacteriological Laboratory, S-105 21 Stockholm, Sweden. reactions to the vaccine and the protective efficacy of the vaccine. The data on side effects and serological efficacy by the neutralisation test have been presented elsewhere. ${ }^{34}$ This report investigates the efficacy of the vaccine in the clinical trial.

\section{Subjects and methods}

From mid-1982 to mid-1983 2 month old infants enrolled at 51 preventive care child health centres in Stockholm who had no known contraindications to pertussis vaccination ${ }^{5}$ were identified to participate in a non-blinded trial. Consent to participate was obtained from all the parents. Infants born on days with an even number were inoculated subcutaneously with three $0.5 \mathrm{ml}$ doses of vaccine given one month apart, starting from the age of 2 months (mean $2 \cdot 3$ (SD 0.5) months, range $1 \cdot 2-7 \cdot 3$ months). Children of the same age born on days with an odd number received no pertussis vaccine. Nurses at the child health centres were instructed to ask parents during the normal regular visits (every 2-4 months) whether the infants in the study had had pertussis and to note any symptoms on a special form kept in the medical records at the centres. Paediatricians at the primary care facilities affiliated with these centres were asked to examine all participants with suspected pertussis and report their findings on a special form.

In February 1985 the report forms of partcipating infants were collected to ascertain the number of cases of pertussis that had occurred in the study population between the ages of 6 and 23 months. In addition, the regular medical records kept at the child health centres were reviewed and the files of laboratory reports to the National Bacteriological Laboratory in Stockholm searched for positive results on culture for children in the study. This laboratory is a governmental agency that receives reports on all positive cultures for pertussis from laboratories throughout Sweden. All samples taken for serological tests from children in the study were identified by a systematic search in the files of the only two laboratories that perform routine serological tests for pertussis. The samples were retested by methods indicated below.

Cases of pertussis were considered to be confirmed by the laboratory if they yielded positive results on culture for Bordetella pertussis or positive serological findings, which were defined as a significant rise in titres in an enzyme linked immunosorbent assay (ELISA) to detect antibodies against filamentous haemagglutinin and pertussis $\operatorname{toxin}^{67}$ or in a neutralisation test in Chinese hamster ovary cells to detect antibodies against pertussis toxin, ${ }^{89}$ or both. $B$ pertussis was cultured in routine laboratories and therefore the serotypes were unknown. The serological assays used were found to have a diagnostic sensitivity in terms of raised titres of $100 \%$ in 90 cases of pertussis that were confirmed by the results of culture (unpublished data).

Cases of pertussis that were not confirmed by laboratory results were included if infants had had typical symptoms such as coughing for more than four weeks, whoops, or coughing with vomiting. In addition, whooping cough had to have been diagnosed by a doctor or have been linked epidemiologically - that is, the infant had been in contact with another patient with whooping cough-or both. These data were supplemented by contacting the parents and the doctors who had diagnosed the disease. 
Statistical analyses of attack rates were performed with corrected $\chi^{2}$ tests and comparison of two independent proportions. Confidence intervals on estimates of the vaccine's efficacy were calculated as described by Orenstein et al. ${ }^{10}$

\section{Results}

Immunisation with three doses of pertussis vaccine from 2 months of age was completed in 525 children. Immunisation was started in 562 infants, but seventeen infants who received only two doses of vaccine and twenty who received only one dose were excluded from the analysis. Altogether 615 children were enrolled as controls.

A total of 55 cases of pertussis occurred among 1140 study participants between the ages of 6 and 23 months (table). Of these, 40 cases $(73 \%)$ were

Attack rate of whooping cough and efficacy of pertussis vaccine by age

\begin{tabular}{|c|c|c|c|c|}
\hline \multirow{2}{*}{$\begin{array}{c}\text { Age group } \\
\text { (months) }\end{array}$} & \multicolumn{2}{|c|}{ Attack rate $(\%)$} & \multicolumn{2}{|c|}{ Efficacy of vaccine (\%) } \\
\hline & Vaccinated group & Unvaccinated group & Estimate & $95 \%$ Confidence interval \\
\hline $6-11$ & $0.4(2 / 525)$ & $3 \cdot 4(21 / 615)$ & $88 \cdot 8$ & $52 \cdot 6$ to $97 \cdot 3$ \\
\hline $12-17$ & $0.6(3 / 523)$ & $2 \cdot 0(12 / 594)$ & $71 \cdot 6$ & 0.0 to 92.3 \\
\hline $18-23$ & $0.6(3 / 520)$ & $2 \cdot 4(14 / 582)$ & $76 \cdot 0$ & $17 \cdot 0$ to $93 \cdot 1$ \\
\hline Total & $1.5(8 / 525)$ & $7 \cdot 6(47 / 615)$ & $80 \cdot 1$ & $58 \cdot 2$ to 90.5 \\
\hline
\end{tabular}

confirmed by the results of culture alone $(n=17)$, the results of culture and serological tests $(n=9)$, or the results of serological tests alone $(n=14)$; in the vaccinated group all eight cases of pertussis were confirmed by laboratory results and in the unvaccinated group the proportion was 32 out of $47(68 \%)$ (the number giving positive results on culture was 5 and 21 , respectively). Of the 15 cases with only a clinical diagnosis, 12 were diagnosed by a doctor and 10 were epidemiologically linked to another case, including the three cases that were not diagnosed by a doctor.

The overall attack rate in the population studied between ages of 6 and 23 months was $4 \cdot 8 \%(55 / 1140)$. The attack rate in vaccinated children was $1 \cdot 5 \%$ $(8 / 525)$ and was significantly lower than that of $7 \cdot 6 \%(47 / 615)$ in unvaccinated children $(p<0.001)$. The estimated efficacy of the vaccine was $80 \%(95 \%$ confidence interval 58 to 90 ) and did not seem to vary between age groups, which corresponded to different lengths of time after vaccination (table). Information on cough and its duration was available for 41 of the 55 infants. Coughing for four weeks or more was noted for two out of six children who were vaccinated and 32 out of 35 who were not vaccinated $(\mathrm{p}<0.001)$ Vaccination with three doses was $93 \%$ ( $95 \%$ confidence interval 72 to 98 ) effective in preventing pertussis with cough duration of four weeks or more.

The attack rate for cases of pertussis confirmed by laboratory results alone-from results of culture or serological results, or both-in vaccinated children was $1 \cdot 5 \%(8 / 525)$ and significantly lower than that of $5 \cdot 2 \%(32 / 615)$ in unvaccinated children $(\mathrm{p}<0.01)$. The estimated efficacy of the vaccine in the cases that were confirmed by laboratory results was thus $71 \%(95 \%$ confidence interval 37 to 86 ), which was similar to that of $72 \%$ (95\% confidence interval 27 to 89 ) for cases confirmed by the results of culture alone.

\section{Discussion}

Few controlled trials of the efficacy of whole cell pertussis vaccine have been conducted since the trials by the British Medical Research Council over 30 years ago. ${ }^{112}$ The situation in Sweden after 1979 provided a unique opportunity to repeat a clinical trial of the efficacy of whole cell vaccine several years after licensure and thereby obtain updated information on this type of pertussis vaccine. Since licensure, numerous reports have estimated the efficacy of the vaccine retrospectively, most commonly from outbreaks of pertussis when attack rates in vaccinated and unvaccinated subjects have been compared. These estimates have, however, varied greatly and it has been difficult to establish the effectiveness of whole cell vaccine. ${ }^{13}$

In our prospective controlled study we allocated subjects by date of birth to the vaccinated and unvaccinated groups and used multiple sources of information to establish cases of pertussis, and a high percentage of our cases were confirmed by laboratory results.
The design of the study should have ensured that the groups were comparable with regard to risk of exposure, and the multiple sources of information should have ensured that all cases of pertussis were properly established. The trial was, however, not blind and despite the many opportunities for a child to be reported to the study as having pertussis evidence exists that all cases of the disease may not have been reported in the unvaccinated group-for example, the percentage of vaccinated children for whom study forms were collected was slightly higher than that for unvaccinated children $(74 \% v 67 \%$, respectively), and review of records was also more complete for the vaccinated children $(78 \% v 66 \%)$. In addition, doctors seemed to seek laboratory confirmation more often for children who were vaccinated; thus serum samples were taken about as often for children who were vaccinated (33 out of 525 ) as for those who were not vaccinated ( 37 out of 615 ), even though the attack rate was higher in the unvaccinated children. This preference by doctors for laboratory confirmation among vaccinated children could explain the fall in the vaccine's estimated efficacy from $80 \%$ for all cases to $71 \%$ for cases with laboratory confirmation alone. A plausible explanation may be that pertussis is a less typical illness among vaccinated children, requiring more laboratory support to be diagnosed. If a bias were introduced by these differences in ascertainment of cases between the groups its direction would be towards artificially low estimates of the vaccine's efficacy.

Our overall estimate of the vaccine's efficacy of $80 \%$ is similar to that found in the trials over 30 years ago. Several problems in interpreting or generalising these results to other populations should be noted. The use of plain rather than adsorbed whole cell vaccine and the lack of a booster injection may explain why our estimate is lower than that observed in some other studies. ${ }^{13}$ Adsorbed pertussis vaccines are currently more commonly used and have been shown in several studies to give a better agglutinin response. ${ }^{14} \mathrm{~A}$ difference in protective efficacy between adsorbed and plain vaccines has not been proved, but studies comparing the two types of vaccine from the same manufacturer have not been large enough to show small differences in efficacy. ${ }^{15}$

Many studies of the agglutinin response after vaccination against pertussis have shown a higher rate of response and higher titres after booster injections than after primary immunisation. ${ }^{\text {is }}$ The antibody response to pertussis toxin (antitoxin) may better reflect long term immunity to disease. ${ }^{9}$ An antitoxin response with this plain whole cell vaccine was elicited after a primary series in about $60 \%$ of the infants in two studies ${ }^{416}$ and in $98 \%$ of a subset of children in our trial, who received a booster injection (unpublished observations). Although the protective efficacy during our study did not decrease significantly, we saw a slight trend in that direction, and the lack of significance may be due to the small numbers.

In conclusion, we obtained an overall estimate of $80 \%$ for the efficacy of a plain whole cell pertussis vaccine given in a primary series of three doses to 2 month old infants. As we found some evidence for selective ascertainment of cases the value obtained may represent an underestimate of the true efficacy of this vaccine and may be lower than that achievable in countries that use a regimen of three doses and one or more booster injections of adsorbed pertussis vaccine.

We thank Gun Rasmuson for her skilful help in compiling the data, and Harald Heijbel for his valuable field work and constructive criticism during the preparation of the manuscript.

\section{References}

1 Granström M, Strandvik B. Kikhostevaccinering av riskgrupper. Lakartidningen 1982;79:1349-50.

2 Eriksson $M$, Granström $M$, Linné $T$. Kikhostevaccination av riskbarn. Lakartidningen 1984;81:36-7.

3 Blennow $M$, Aurelius G, Olin P. Biverkningsrapport från klinisk prövning av engelskt kikhostevaccin på spädbarn. Lakartidningen 1984;81:2905-6.

4 Granström M. Discussion. Dev Biol Stand 1985;61:379-86.
5 Anonymous. Whooping-cough immunisation [Editorial]. Br Med f 1977;ii:5-6.

6 Granström M, Lindberg AA, Askelöf P, Hederstedt B. Detection of antibodies in human serum Granstrom $M$, Lindberg AA, Askelof $P$, Hederstedt $B$. Detection of antibodies in human serum
against fimbrial haemagglutinin of Bordetella pertussis by enzyme-linked immunosorbent assay. 7 Med Microbiol 1982;15:85-96.

7 Granström $M$, Granström $G$, Lindfors A, Askelöf $P$. Serologic diagnosis of whooping cough by an 
enzyme-linked immunosorbent assay using fimbrial haemagglutinin as antigen. $\mathcal{f}$ Infect $D$ is 1982;146:741-5.

8 Gillenius $P$, Jäätmaa E, Askelöf $P$, Granström $M$, Tiru $M$. The standardization of an assay for pertussis toxin and antitoxin in microplate culture of Chinese hamster ovary cells. $f$ Biol Stand 1985;13:61-6.

9 Granström $M$, Granström G, Gillenius $P$, Askelöf $P$. Neutralizing antibodies to pertussis toxin in whooping cough. $\mathcal{F}$ Infect Dis $1985 ; 151: 646-9$.

10 Orenstein WA, Bernier RH, Dondero TJ, et al. Field evaluation of vaccine efficacy. Bull WHO 1985;63:1055-68.

11 Medical Research Council. The prevention of whooping-cough by vaccination. Br Med $\mathcal{J}$ 1951;i:1463-71.

12 Laurell G, Mellbin T, Rabo E, Vahlquist B, Zetterquist P. Systematische Impfung mit kombiniertem Impfstoff (Diphtherie, Tetanus, Pertussis) im Säuglingsalter. Serologische und klinische Studien. Klin Wochenschr 1957;35:920-4.
13 Cherry JD. The epidemiology of pertussis and pertussis immunization in the United Kingdom and the United States: a comparative study. Curr Probl Pediatr 1984;14:1-78.

14 Preston NW, Mackay RI, Bamford FN, Crofts JE, Burland WL. Pertussis agglutinins in W vaccinated children: better response with adjuvant. foumal of Hygiene (Cambridge) 1974;73:119-25.

15 Butler NR, Wilson BDR, Benson PF, Dudgeon JA, Ungar J, Beale AJ. Response of infants to pertussis vaccine at one week and to poliomyelitis, diphtheria, and tetanus vaccine at six $C$ months. Lancet 1962;ii:112-4. Blennow $M$, Granstom

Preliminary data from a clinical trial (phase 2) of an acellular pertussis vaccine, J-NIH-6. Dev Biol Stand 1986;65:185-90.

(Accepted 26 fanuary 1988

\section{SHORT REPORTS}

\section{Small bowel volvulus in two siblings}

We report two cases of small bowel volvulus occurring in brothers, one with tragic consequences. Cases of intestinal atresia, probably as a result of intrauterine volvulus, have been reported in siblings, ${ }^{12}$ but volvulus alone has not.

\section{Case reports}

Case 1-A $4 \frac{1}{2}$ year old boy presented with a three hour history of vomiting, abdominal pain, and drowsiness. He had previously been well, and his medical history was unremarkable. On examination he was not feverish but was tachycardic, tachypnoeic, and peripherally shut down. His abdomen was soft and not obviously tender or distended. Before much investigation had been carried out, however, his condition deteriorated further, and in spite of vigorous attempts at resuscitation he died. Postmortem examination showed that virtually all of the small intestine was ischaemic, with twisting of the root of the mesentery. The cause of death was recorded as volvulus of the small bowel.

Case 2-The younger brother of the patient in case 1 was referred to this hospital at the age of 4 with a history of two episodes of sudden abdominal pain associated with profuse vomiting. These episodes settled quickly without treatment, and no haematological or radiological abnormalities were found when he was examined, although his symptoms had subsided by this time. Because his parents were naturally worried that the symptoms were caused by small bowel volvulus, as in their first son, a laparotomy was performed. This was decided on because even if other investigations-for example, barium enema or barium meal and follow through - had yielded normal results the parents would not have been reassured until a laparotomy had been performed. Surprisingly, at laparotomy the bowel was found to be malrotated and the small bowel to be suspended on a long, narrow based mesentery, which showed signs of previous episodes of volvulus. The malrotation was treated, and he made a good recovery, experiencing no further problems.

\section{Comment}

Malrotation of the small intestine is caused by disordered movement of the intestine around the superior mesenteric artery during embryological development. ${ }^{3}$ Two main abnormalities that produce clinical syndromes may occur. In the first narrowing of the base of the mesentery occurs, which may allow the midgut to twist and cause a volvulus. This may occur acutely, causing complete obstruction, or intermittently, producing bouts of obstruction that resolve spontaneously. In the second Ladd's bands are present, which rarely cause acute symptoms.

Malrotation can cause symptoms at any age, but most cases are seen in childhood. Most children who develop symptoms related to volvulus do so within the neonatal period, presenting with features of intestinal obstruction. Unless the obstruction is partial persistent vomiting occurs from the onset. The vomitus may or may not contain bile. Abdominal distension is often absent. ${ }^{4}$ Blood in the stool or vomit is a serious sign of impending bowel infarction. Features in plain abdominal radiographs vary, but duodenal dilatation and malposition of bowel loops are usually present. Dehydration occurs rapidly, and resuscitation and early laparotomy are essential. Children with malrotation who present later in childhood may do so with features of intermittent obstruction, such as intermittent episodes of vomiting and abdominal pain, ${ }^{3}$ as in case 2 .

Laparotomy is indicated when symptoms occur, and ischaemic bowel may need resection. When ischaemia is extensive resection may not be feasible, and after the volvulus has been untwisted a second investigatory operation should be considered. Ladd's operation (or one of its various modifications) is usually the procedure of choice.

Volvulus during intrauterine life may cause intestinal atresia, which has been reported in siblings. ${ }^{12}$ In the "apple peel syndrome" there is duodenal or high jejunal atresia, and an autosomal recessive type of inheritance has $\overrightarrow{0}$ been postulated. Volvulus occurring in infants without atresia has not previously been reported in siblings.

The outcome in the first case was most unfortunate, but the parents' wish to have a laparotomy performed on their second son may have saved him from the same fate.

1 Mishalany HG, Najjar FB. Familial jejunal atresia: three cases in one family. $J$ Pediatr 1968;73 753-5.

Dickson JAS. Apple peel small bowel: an uncommon variant of duodenal and jejunal atresia. J Pediatr Surg 1970;5:595-600.

3 Walker-Smith JA, Wright V. Congenital abnormalities of the gastrointestinal tract. In: Weatherall DJ, Ledingham JGG, Warrell DA, eds. Oxford textbook of medicine. Vol 1. Oxford: Oxford University Press, 1983:12-144.

4 Atwell JD. Pitfalls in the diagnosis of intestinal obstruction in the newborn. Proc $R$ Soc Med 1971;64:26-9.

5 Cathcart RS, Williamson B, Gregorie HB. Surgical treatment of midgut non-rotation in the adult patient. Surg Gynecol Obstet 1981;152:207-10.

(Accepted 16 February 1988)

Department of Surgery, Princess Margaret Hospital, Swindon, Wilts SN1 4JU

J S BUDD, FRCS, registrar

P H POWLEY, FRCS, consultant surgeon

Correspondence to: Mr J S Budd, University Department of Surgery, Leicester Royal Infirmary, Leicester LE1 5WW.

\section{Effect of topical corticosteroids on seasonally induced increases in nasal mast cells}

Symptoms of allergic rhinitis caused by grass pollen become more severe as the pollen season progresses despite falling pollen counts, a phenomenon known as "nasal priming."

We have shown that there is a substantial and significant increase in the $N$ density of mast cells in the nasal mucosa of patients who have seasonal $D$ allergic rhinitis in summer compared with that in winter, ${ }^{2}$ which would facilitate an interaction between cell bound immunoglobulin and allergen $\mathrm{N}$ and offer an explanation for nasal priming. The topical administration of corticosteroids is an established and effective treatment for allergic rhinitis, but its mode of action remains unknown. We investigated the effect of a topical corticosteroid, beclomethasone dipropionate, on seasonally induced increases in the density of mast cells.

\section{Patients, methods, and results}

Nine men and nine women (mean age 23.7 years) were studied. All had a five year history of seasonal rhinitis and gave positive results to skin prick tests with mixed grass pollen (2.5\% weight/volume) and negative results to skin prick tests with house dust $(150 \%$ weight/volume $)$ and house dust mite $(1 \cdot 2 \%$ weight/volume.) A biopsy sample was taken from the inferior turbinate of each patient during the first week of March 1986 before the grass pollen season started. Patients were randomised (with a random number table) in May 1986 to receive double blind treatment with beclomethasone dipropionate (Allen and Hanburys Ltd, Greenford, UK) or placebo $100 \mu \mathrm{g}$ twice a day to each nostril from identical 